\title{
Relationship of melatonin level, oxidative stress and inflammatory status with osteoporosis in maintenance hemodialysis of chronic renal failure
}

\author{
HAIWEI REN ${ }^{1 *}$, RENLIAN SUN ${ }^{1 *}$ and JIAN WANG ${ }^{2}$ \\ Departments of ${ }^{1}$ Dialysis and ${ }^{2}$ Nephrology, Dezhou People's Hospital, Dezhou, Shandong 253014, P.R. China
}

Received July 28, 2017; Accepted November 29, 2017

DOI: $10.3892 / \mathrm{etm} .2018 .5857$

\begin{abstract}
The relationship of melatonin (MT) level, oxidative stress and inflammatory status with osteoporosis in the maintenance hemodialysis (MHD) of chronic renal failure was investigated. Ninety-four patients with chronic renal failure treated in Dezhou People's Hospital (Dezhoo, China) and receiving MHD for more than 3 months from May to April 2017 were selected and divided into the osteoporosis group (observation group, $\mathrm{n}=49$ ) and the non-osteoporosis group (control group, $n=45$ ) based on whether osteoporosis was involved. The serum advanced oxidation protein products (AOPP), MT level and inflammatory factors [tumor necrosis factor- $\alpha$ (TNF- $\alpha$ ), interleukin-6 (IL-6) and IL-1] in patients were detected via enzyme-linked immunosorbent assay (ELISA). Moreover, the malondialdehyde (MDA) level in serum was detected via thibabituric acid (TBA) assay and the spinal bone mineral density (BMD) was detected using the total BMD testing instrument, followed by correlation analysis. The MT level in observation group was significantly lower than that in control group, but the levels of AOPP and MDA were significantly higher than those in control group $(\mathrm{P}<0.05)$. The levels of inflammatory factors (TNF- $\alpha$, IL-6 and IL-1) in observation group were obviously higher than those in the control group $(\mathrm{P}<0.05)$. Besides, the spinal BMD and T-value in observation group were significantly lower than those in the control group $(\mathrm{P}<0.05)$. Pearson's correlation analysis showed that AOPP, MDA, TNF- $\alpha$, IL- 6 and IL-1 levels were negatively correlated with $\mathrm{BMD}$, but MT was positively correlated with BMD $(\mathrm{P}<0.05)$. Osteoporosis occurs easily in MHD patients; oxidative stress and inflammatory degree are
\end{abstract}

Correspondence to: Dr Jian Wang, Department of Nephrology, Dezhou People's Hospital, 1751 Xinhu Street, Dezhou, Shandong 253014, P.R. China

E-mail:nhlw22w@126.com

*Contributed equally

Key words: chronic renal failure, maintenance hemodialysis, melatonin, oxidative stress, inflammation, osteoporosis negatively correlated with osteoporosis, but MT is positively correlated with osteoporosis.

\section{Introduction}

Osteoporosis (OP) is a clinically common disease in systemic skeletal system, characterized by the microstructural damage of bone tissues and low bone mass, and patients are prone to fracture (1). Maintenance hemodialysis (MHD) of chronic renal failure (CRF) will lead to a variety of complications, such as infection, cardiovascular diseases and OP. The OP incidence rate in MHD patients is up to 40-60\%, seriously affecting their life quality (2). MHD patients often have oxidative stress (OS) and microinflammatory status; advanced oxidized protein product (AOPP) is one of the markers reflecting the OS status in the body, and malondialdehyde (MDA) is the end metabolite of OS and can be used as a marker reflecting the OS level in the body; the microinflammation in MHD patients is often characterized by the increased tumor necrosis factor- $\alpha$ (TNF- $\alpha$ ), interleukin-6 (IL-6) and IL-1 $(3,4)$. Melatonin (MT) is a kind of hormone secreted by the pineal gland, which not only has the effects of anti-inflammation, anti-oxidation and wakening regulation, but also can promote the proliferation and differentiation of osteoblasts and inhibit the proliferation and differentiation of osteoclasts through a variety of ways, and it has a close relationship with OP (5). In this study, the levels of MT, AOPP, MDA, TNF- $\alpha$, IL- 6 and IL-1 in CRF patients receiving MHD were detected to analyze whether they were involved in the occurrence of OP and their relationships with osteoporosis.

\section{Materials and methods}

General materials. Ninety-four CRF patients treated in Dezhou People's Hospital (Dezhoo, China) from May 2016 to April 2017 were selected. Inclusion criteria: 1) patients meeting the diagnostic criteria of chronic renal failure; 2) patients receiving MHD for more than 3 months; 3) patients who signed the informed consent. Exclusion criteria: 1) patients with severe heart, brain or liver diseases, or accompanied with mental illness; 2) patients with malignant tumors, pregnant or lactating women. According to whether osteoporosis was involved, patients enrolled were divided into the osteoporosis group (observation group, $n=49$ ) and the non-osteoporosis 
Table I. Baseline data of patients in the two groups.

\begin{tabular}{lcccr}
\hline Item & Control group $(\mathrm{n}=45)$ & Observation group $(\mathrm{n}=49)$ & $\mathrm{t} / \chi^{2}$ test & P-value \\
\hline Sex (male/female) & $25 / 20$ & $29 / 20$ & 0.022 & 0.883 \\
Age (years) & $35-70$ & $35-65$ & & \\
Average age (years) & $46.56 \pm 8.43$ & $46.85 \pm 8.52$ & 0.166 & 0.869 \\
Course of disease (month) & $13.62 \pm 3.46$ & $13.25 \pm 3.37$ & 0.525 & 0.601 \\
BMI $\left(\mathrm{kg} / \mathrm{m}^{2}\right.$ ) & $22.43 \pm 3.27$ & $21.87 \pm 3.58$ & 0.790 & 0.432 \\
Blood glucose (mmol/l) & $5.78 \pm 1.15$ & $5.63 \pm 1.24$ & 0.607 & 0.545 \\
Total cholesterol (mmol/l) & $6.12 \pm 0.73$ & $6.09 \pm 0.68$ & 0.206 & 0.837 \\
Triglyceride (mmol/l) & $2.38 \pm 1.27$ & $2.52 \pm 1.34$ & 0.519 & 0.605 \\
\hline
\end{tabular}

group (control group, $n=45$ ). There were no statistically significant differences in the baseline data between the two groups $(\mathrm{P}>0.05)$ (Table I).

Hemodialysis method. Using the hemodialysis machine (Fresenius, Oberursel, German), the double-lumen catheter was placed in the right side of the neck or right femoral vein to establish cardiopulmonary bypass; the diluted displacement liquid (Shanghai Changzheng Pharmaceutical Co., Shanghai, China) was injected using the negative-pressure ultra-filter pump, and the low-molecular-weight heparin (Tianjin Chase Sun Pharmaceutical Co., Ltd., Tianjin, China, approval no. NMPN H20020469) was used for anticoagulation (first dose 3,000 U, addition $500 \mathrm{U} / \mathrm{h}$, replacement rate $4.0 \mathrm{l} / \mathrm{h}$ ), and the blood flow was maintained at approximately $200 \mathrm{ml} / \mathrm{min}$. Patients were treated for $4 \mathrm{~h}$ each time and received dialysis 3 times per week.

Bone mineral density (BMD) detection. BMD was measured using dual-energy X-ray bone densitometer (Shenzhen XRAY Electric Co., Ltd., Shenzhen, China), including the lateral lumber spine. The graphical analysis area was divided into total lateral lumber spine and lateral medial area (approximately $1 / 2$ of the whole vertebral body in the length and width); the T-value (the ratio of patients' BMD to age) was calculated.

Detection of MT, AOPP, MDA, TNF- $\alpha, I L-6$ and IL-1 levels. Venous blood $(5 \mathrm{ml})$ was collected from patients (at 7:00 in the morning after fasting for $8 \mathrm{~h}$ ), and the serum was immediately separated and placed at $-80^{\circ} \mathrm{C}$ for standby application. The levels of serum AOPP, MT, TNF- $\alpha$, IL- 6 and IL- 1 were measured via enzyme-linked immunosorbent assay (ELISA). The relevant kits were provided by Shanghai Hengyuan Biotechnology Co., Ltd. (Shanghai, China) and the operation was strictly according to the instructions of the kit. Samples (1-fold dilution) and $100 \mu \mathrm{l}$ enzyme-labeled solution were added into the micro-holes of reaction plate for incubation at $37^{\circ} \mathrm{C}$ for $90 \mathrm{~min}$. After the plate was washed 3 times $(15 \mathrm{sec}$ interval at every two times), the color developing agents $\mathrm{A}$ and $\mathrm{B}$ (50 $\mu \mathrm{l}$ for each) were added for incubation at room temperature at $20^{\circ} \mathrm{C}$ in the dark for $15 \mathrm{~min}$. The $\mathrm{OD}$ value was read at a wavelength of $450 \mathrm{~nm}$ using the microplate reader (Shenzhen SinoThinker Technology Co., Ltd., Shenzhen, China) within 15 min. Then the levels of AOPP, MT, TNF- $\alpha$, IL-6 and IL-1 were calculated.
The serum MDA was detected via thiobarbituric acid (TBA) assay. Preparations: the reagent $\mathrm{A}$ in the MDA kit (Nanjing Jiancheng Bioengineering Institute, Nanjing, China) was heated to transparency through water bath, and $340 \mathrm{ml}$ double distilled water was added and mixed evenly into the reagent $\mathrm{B}$ and stored at $4^{\circ} \mathrm{C}$. After the reagent $\mathrm{C}$ was fully dissolved by $60 \mathrm{ml}$ double distilled water $\left(95^{\circ} \mathrm{C}\right), 60 \mathrm{ml}$ glacial acetic acid was added, mixed evenly and stored in the dark at $4^{\circ} \mathrm{C}$. The standard product $(10 \mathrm{nmol} / \mathrm{ml}$ tetraethoxypropane) was stored at $4^{\circ} \mathrm{C}$. Operating steps: 1) The standard product $(1 \mathrm{ml})$ was added into the standard tube, the serum $(0.1 \mathrm{ml})$ was added into the testing tube, and the anhydrous ethanol was added into the standard blank tube; 2$)$ the reagent $\mathrm{A}(0.1 \mathrm{ml})$ was added and mixed evenly into the standard tube, testing tube, standard blank tube and testing blank tube, and then the reagent $\mathrm{B}(0.1 \mathrm{ml})$ was added; 3$)$ the reagent $\mathrm{C}(0.1 \mathrm{ml})$ was added into the standard tube, testing tube and standard blank tube, and $50 \%$ glacial acetic acid $(0.1 \mathrm{ml})$ was added into the testing blank tube; 4) the solution was mixed evenly using the swirling mixer; the tube mouth was tightened using the fresh-keeping membrane and one riser vent was punctured; then the tube was soaked in hot water $\left(95^{\circ} \mathrm{C}\right)$ for $40 \mathrm{~min}$ and cooled under the running water; 5) after the centrifugation for $10 \mathrm{~min}$ at 2,000 $\mathrm{xg}$, the supernatant was taken, the OD value at a wavelength of $532 \mathrm{~nm}$ was measured using the microplate reader, and the content of MDA in serum was calculated.

Evaluation criteria. Fasting venous blood (5 ml) was collected from patients in the morning. The levels of serum AOPP, inflammatory factors (TNF- $\alpha$, IL-6 and IL-1) and MT were analyzed via ELISA, and the level of MDA was detected via TBA assay.

Judgment criteria of OP: 1) normal bone mass: T-value $>-1$; 2) bone mass loss: T-value $=-1$ to -2 ; 3) OP: T-value $<-2$.

Statistical analysis. Data were processed using SPSS 19.0 software (SPSS Inc., Chicago, IL, USA). Measurement data are presented as mean \pm standard deviation, and t-test was used; enumeration data are presented as ratio, and Chi-square test was used. Pearson's correlation coefficient analysis was used for the correlation. $\mathrm{P}<0.05$ was considered to indicate a statistically significant difference. The study was approved by the Ethics Committee of Dezhou People's Hospital and written informed consents were signed by the patients and/ or guardians. 
Table II. Comparisons of oxidative stress and MT level in patients between the two groups.

\begin{tabular}{lcccc}
\hline Group & $\mathrm{n}$ & $\begin{array}{c}\text { AOPP } \\
(\mu \mathrm{mol} / \mathrm{l})\end{array}$ & $\begin{array}{c}\mathrm{MDA} \\
(\mathrm{nmol} / \mathrm{l})\end{array}$ & $\begin{array}{c}\mathrm{MT} \\
(\mathrm{pg} / \mathrm{ml})\end{array}$ \\
\hline Observation group & 49 & $108.95 \pm 15.13$ & $5.97 \pm 1.47$ & $19.76 \pm 3.38$ \\
Control group & 45 & $43.69 \pm 9.06$ & $2.73 \pm 0.86$ & $24.43 \pm 3.27$ \\
t-test & & 25.091 & 12.894 & 6.797 \\
P-value & & $<0.001$ & $<0.001$ & $<0.001$ \\
\hline
\end{tabular}

AOPP, advanced oxidation protein products; MDA, malondialdehyde; MT, melatonin.

Table III. Comparisons of inflammatory factor levels in patients between the two groups (ng/l).

\begin{tabular}{lcccc}
\hline Group & $\mathrm{n}$ & TNF- $\alpha$ & $\mathrm{IL}-6$ & $\mathrm{IL}-1$ \\
\hline Observation group & 49 & $49.98 \pm 3.15$ & $53.48 \pm 3.35$ & $47.28 \pm 3.19$ \\
Control group & 45 & $37.83 \pm 3.04$ & $41.45 \pm 3.23$ & $35.39 \pm 3.17$ \\
t-test & & 18.996 & 17.693 & 18.106 \\
P-value & $<0.001$ & $<0.001$ & $<0.001$ \\
\hline
\end{tabular}

TNF, tumor necrosis factor; IL, interleukin.

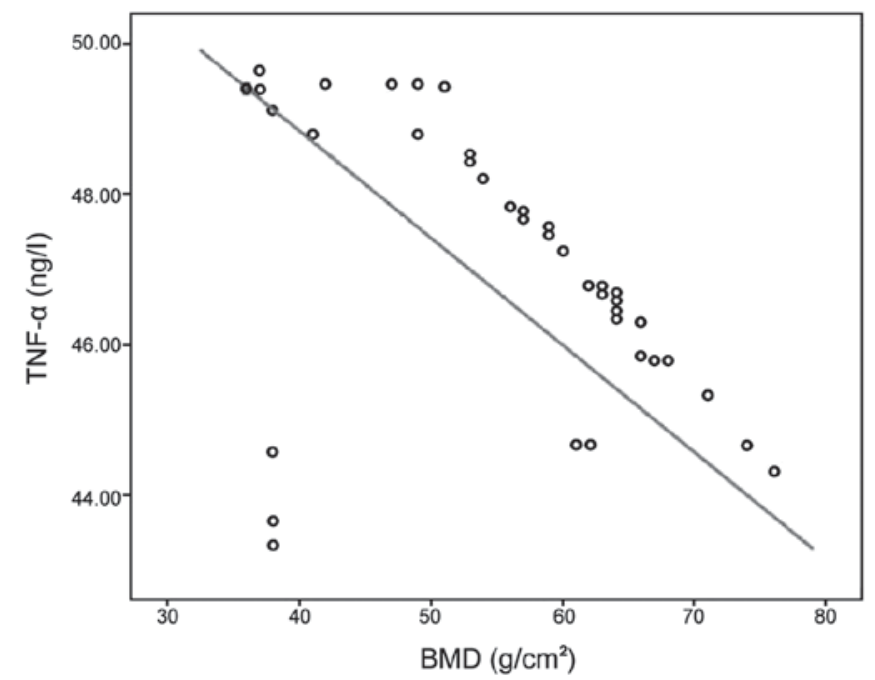

Figure 1. Correlation analysis of tumor necrosis factor (TNF)- $\alpha$ level with bone mineral density (BMD).

\section{Results}

Comparison of oxidative stress and MT level in patients in the two groups. The levels of AOPP and MDA in observation group were significantly higher than those in the control group, but MT level was significantly lower than that in the control group $(\mathrm{P}<0.05)($ Table II).

Comparisons of inflammatory factor levels in patients in the two groups. The levels of TNF- $\alpha$, IL-6 and IL-1 in observation

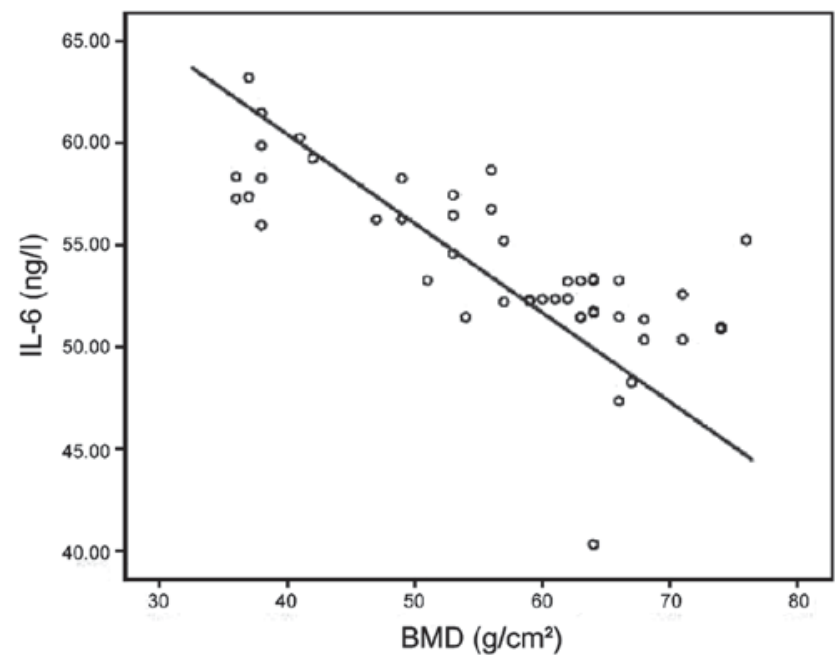

Figure 2. Correlation analysis of interleukin (IL)-6 level with bone mineral density (BMD).

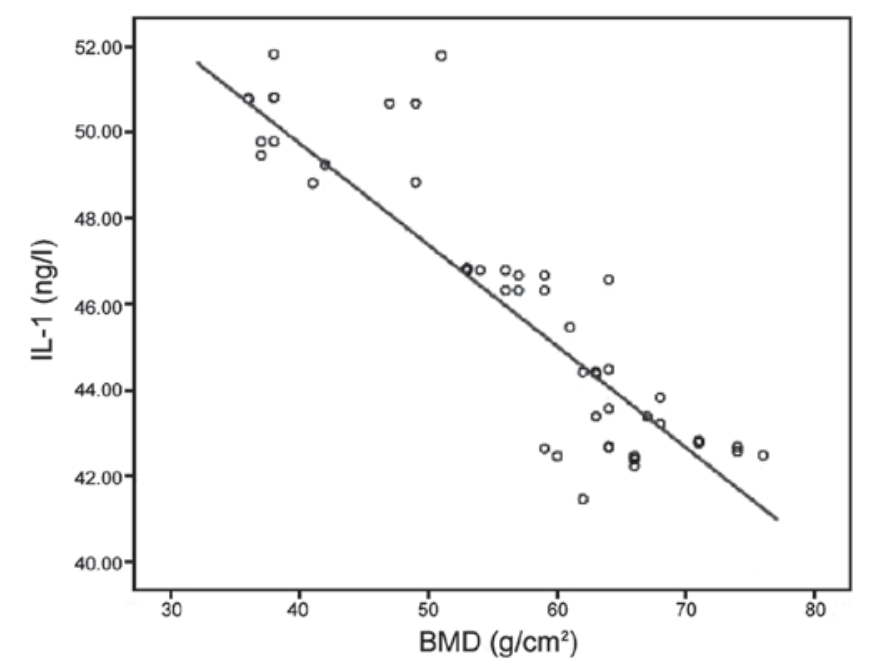

Figure 3. Correlation analysis of interleukin (IL)-1 level with bone mineral density (BMD).

group were obviously higher than those in control group $(\mathrm{P}<0.05)$ (Table III).

Comparisons of mid-lateral lumbar vertebrae and total lumbar BMD. The mid-lateral lumbar vertebrae and total lumbar BMD and T-values in observation group were significantly lower than those in control group $(\mathrm{P}<0.05)$ (Table IV).

Correlation analysis of oxidative stress, inflammation and $M T$ level with BMD. Pearson's correlation analysis showed that AOPP, MDA, TNF- $\alpha$, IL- 6 and IL-1 levels were negatively correlated with BMD, but MT was positively correlated with BMD $(\mathrm{P}<0.05)$ (Figs. 1-6, Table V).

\section{Discussion}

OP is caused by the bone metabolic disorders (bone resorption is greater than bone formation), characterized by the decreased 
Table IV. Comparisons of lateral lumbar bone mineral density (BMD) and T-value.

\begin{tabular}{lcccccc}
\hline & & \multicolumn{2}{c}{$\mathrm{BMD}\left(\mathrm{g} / \mathrm{cm}^{2}\right)$} & & \multicolumn{2}{c}{ T-value } \\
Group & $\mathrm{n}$ & Total lateral lumbar & Mid-lateral lumbar & & Total lateral lumbar & Mid-lateral lumbar \\
\hline Observation group & 49 & $0.73 \pm 0.12$ & $0.65 \pm 0.13$ & & $-3.75 \pm 0.93$ & $-4.15 \pm 0.86$ \\
Control group & 45 & $0.91 \pm 0.13$ & $0.86 \pm 0.14$ & & $-1.03 \pm 0.86$ & $-1.09 \pm 0.76$ \\
t-test & 6.981 & 7.541 & & 14.683 & 18.213 \\
P-value & $<0.001$ & $<0.001$ & & $<0.001$ & $<0.001$ \\
\hline
\end{tabular}

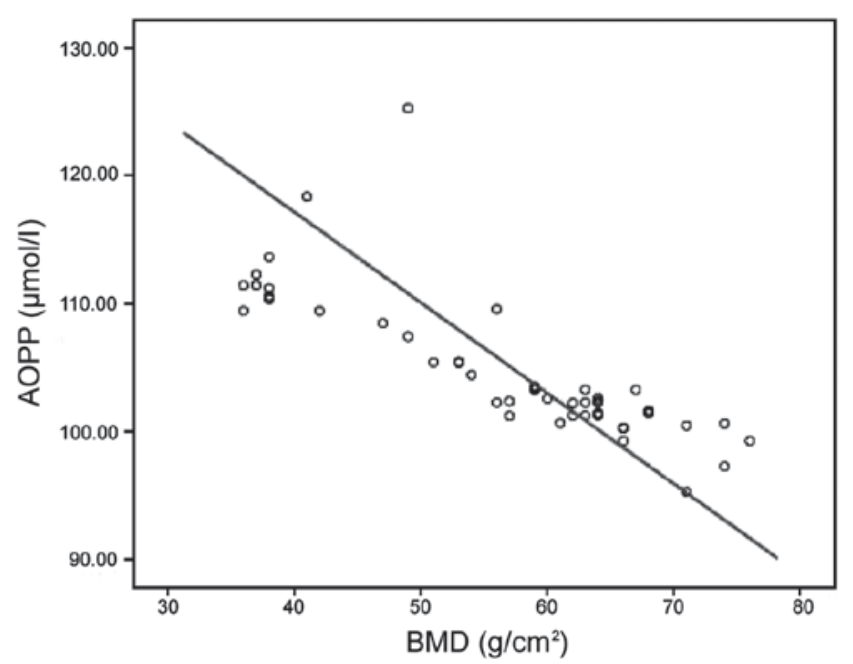

Figure 4. Correlation analysis of advanced oxidation protein products (AOPP) level with bone mineral density (BMD).

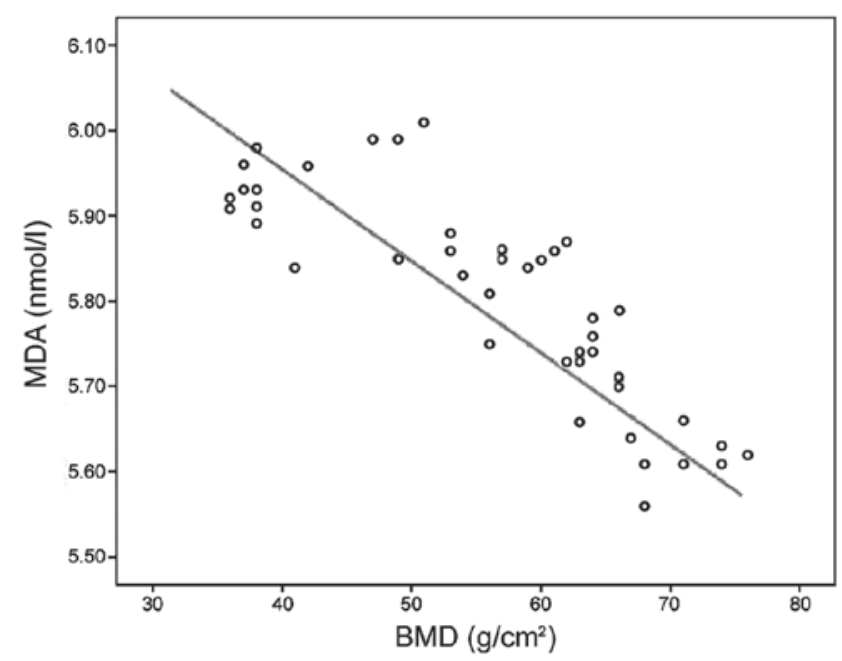

Figure 5. Correlation analysis of malondialdehyde (MDA) level with bone mineral density (BMD).

bone mass per unit volume and increased bone fragility, and it will continuously develop and easily leads to fractures (6). In the past, it was thought that the mechanism of OP accompanied in CRF patients was that the calcium-phosphorus metabolic disturbance in patients reduced the level of vitamin D in the body, thus leading to metabolic acidosis and secondary hyperparathyroidism. In recent years, with the deepening of

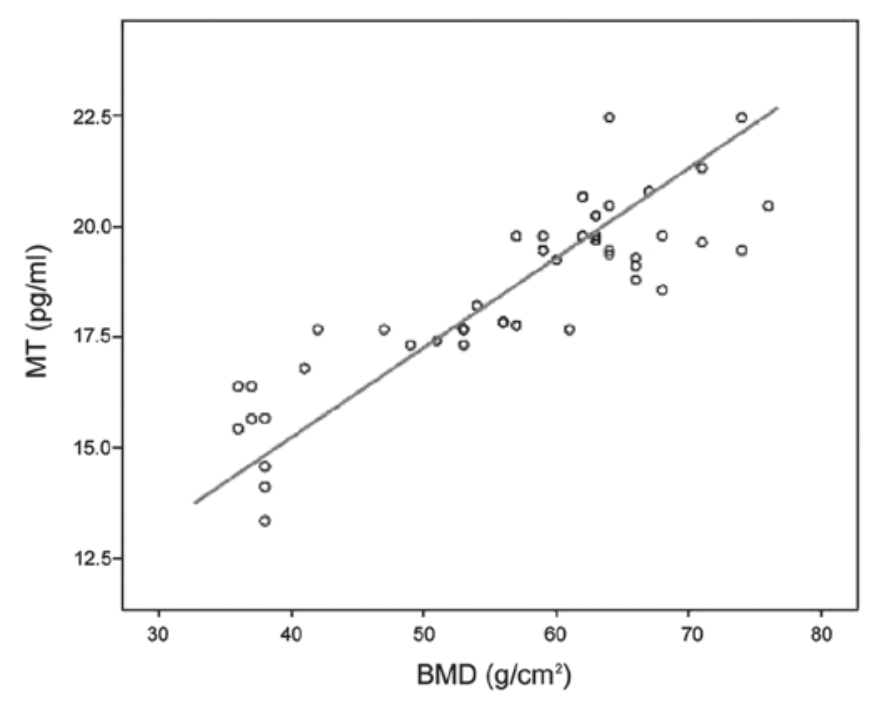

Figure 6. Correlation analysis of melatonin (MT) level with bone mineral density (BMD).

Table V. Correlation analysis of oxidative stress, inflammation and MT level with BMD.

\begin{tabular}{lllllr}
\hline & \multicolumn{5}{c}{ Correlation with BMD } \\
\cline { 2 - 6 } Item & r-value & P-value & Item & r-value & P-value \\
\hline TNF- $\alpha$ & -0.487 & -0.401 & AOPP & -0.406 & -0.424 \\
IL-6 & -0.401 & -0.417 & MDA & -0.424 & 0.439 \\
IL-1 & -0.417 & -0.406 & MT & 0.439 & 0.015
\end{tabular}

$\mathrm{BMD}$, bone mineral density; AOPP, advanced oxidation protein products; MDA, malondialdehyde; MT, melatonin.

research, it has been recognized that the bone metabolism in CRF patients receiving long-term MHD will be jointly regulated by a variety of hormones and cytokines, thus causing the abnormal expression levels and functions of OPG/RANKL/ RANK system in the body, ultimately affecting the differentiation and function of osteoclasts and inducing OP (7).

MT is a kind of indole neuroendocrine hormone secreted by pineal body, as well as the strongest free radical scavenger currently known (8). Studies have shown that MT secretion in CRF patients is significantly lower than that in normal population, especially in MHD patients whose MT peak at 
night disappears (9). The antioxidant mechanism in normal state of human body is perfect, which can eliminate oxygen free radicals through the enzyme catalysis and enzyme-free catalysis, thus playing an antioxidant effect (10). When the defense system function in the body is low, the redox reaction will be out of balance, resulting in the accumulation of reactive oxygen species (ROS), imbalance between oxidation and antioxidation, among which oxidation dominates, causing OS (11). AOPP is a kind of double-arginine protein cross-linking agent produced by albumin in OS state. MDA is a toxic substance produced by the peroxidation of unsaturated fatty acids in the cell membrane due to superoxide anion in the body $(12,13)$. The results of this study showed that the level of MT in observation group was significantly lower than that in control group, but the levels of AOPP and MDA were significantly higher than those in the control group $(\mathrm{P}<0.05)$, indicating that CRF patients with OP are in an obvious oxidative-antioxidant system imbalance, because AOPP results not only from ROS, but also is the result of OS. Besides, MDA has a strong cytotoxicity and will cause the cross-linking polymerization of macromolecules (proteins, lipids and nucleic acids), whose content directly reflects the rate and intensity of lipid peroxidation, and it can be used as an index for the severity of free radical damage, indirectly reflecting the process of oxygen free radical damage. The decreased MT level may be related to OS, and interacts in CRF patients with OP.

Clinically, BMD is often used for the OP diagnosis. Cancellous bone $(60-75 \%)$ in the total skeletal system is concentrated in the human lumbar spine, so the measurement of lumbar BMD in MHD patients can reflect the early changes in bone mineral content. In addition, the bone turnover rate of cancellous bone is higher than that of cortical bone, and it is more sensitive to all kinds of metabolic stimuli. Moreover, its accumulation of bone mass will reach the peak earlier than cortical bone, so the measurement of lateral lumbar BMD has a higher sensitivity (14).

The proteinuria and high renin - angiotensin activity, in CRF patients can stimulate the body's inflammatory response, and inhibit the $\mathrm{T}$ lymphocyte immune function and lead to immune dysfunction (15). TNF- $\alpha$ is an important mononuclear inflammatory factor, and IL-6 is a member of the interleukin family, as well as a lymphocyte factor in the acute-phase response, playing a variety of roles in the immune response (16). Moreover, IL-1 is also one of the important members of the interleukin family, including IL-1 $\alpha$ and IL- $1 \beta$, in which IL- $1 \beta$ is one of the important inflammatory cytokines in the inflammatory process (17). The results of this study showed that the levels of TNF- $\alpha$, IL-6 and IL-1 in observation group were obviously higher than those in control group $(\mathrm{P}<0.05)$, suggesting that the microinflammation is more obvious in CRF patients with OP.

The results in this study showed that AOPP, MDA, TNF- $\alpha$, IL-6 and IL-1 levels were negatively correlated with BMD, but MT was positively correlated with BMD $(\mathrm{P}<0.05)$, because enough MT will decrease the formation and activation of osteoclasts, thereby inhibiting the bone resorption and increasing the bone mass (18). In addition, MT can induce the secretion of growth hormone, and its synergistic application can promote the formation of new bones. But with the extension of MHD, the patients are prone to pineal body calcification; and the greater the area of calcification is, the less the MT will be secreted and the more easily the patient will be prone to OP, suggesting that MT is negatively correlated with OP. Inflammatory factors, such as TNF- $\alpha$, IL-6 and IL-1, usually exhibit the effect of promoting bone resorption and inhibiting bone formation; in particular, TNF- $\alpha$ can induce the stromal cell expression in osteoblasts and stimulate the osteoclast activation. IL-1 can promote the osteoclast precursor cell proliferation and differentiation and enhance the osteoclast activity, making the bone resorption greater than bone formation and inducing OP (19). When the oxidantantioxidant function is unbalanced in the body, it will inhibit the differentiation of osteoblast precursor cells into osteoblasts, inhibit the mineralization of osteoblasts and induce its death. AOPP inhibits the proliferation and differentiation of osteoblastlike cells through activating the $N F-\kappa B$ pathway. Besides, MDA can inhibit the protein and nucleic acid synthesis and decrease the enzyme activity, resulting in decreased antioxidant substances in the body and thus gradually reducing the bone mass. After OS stimulates the osteoclast growth and differentiation, osteoclasts will in turn produce more ROS, exacerbate the damage to the antioxidant defense mechanism in the body and form a vicious circle, ultimately leading to extensive OP (20).

In conclusion, MHD patients are prone to OP, and there are significant OS and inflammatory status; the MT level, OS and inflammatory degree in patients are closely related to OP. The clinical exploration of their relationship is of great significance in reducing the incidence rate of OP in $\mathrm{CRF}$ patients receiving MHD.

\section{Acknowledgements}

Not applicable.

\section{Funding}

No funding was received.

\section{Availability of data and materials}

The datasets used and/or analyzed during the current study are available from the corresponding author on reasonable request.

\section{Authors' contributions}

HR and RS detected bone mineral density and MT, AOPP, MDA, TNF- $\alpha$, IL-6 and IL-1 levels. JW performed thiobarbituric acid assay. All authors read and approved the final manuscript.

\section{Ethics approval and consent to participate}

The study was approved by the Ethics Committee of Dezhoo People's Hospital (Dezhoo, China). Written informed consents were signed by the patients and/or guardians.

\section{Consent for publication}

Not applicable.

\section{Competing interests}

The authors declare that they have no competing interests. 


\section{References}

1. Jeremiah MP, Unwin BK, Greenawald MH and Casiano VE: Diagnosis and management of osteoporosis. Am Fam Physician 92: 261-268, 2015.

2. Shigematsu T, Muraoka R, Sugimoto T and Nishizawa Y: Risedronate therapy in patients with mild-to-moderate chronic kidney disease with osteoporosis: Post-hoc analysis of data from the risedronate phase III clinical trials. BMC Nephrol 18: 66, 2017.

3. Del Vecchio L, Locatelli F and Carini M: What we know about oxidative stress in patients with chronic kidney disease on dialysis - clinical effects, potential treatment, and prevention. Semin Dial 24: 56-64, 2011.

4. Oh DJ, Kim HR, Lee MK and Woo YS: Profile of human $\beta$-defensins 1,2 and proinflammatory cytokines (TNF- $\alpha$, IL-6) in patients with chronic kidney disease. Kidney Blood Press Res 37: 602-610, 2013

5. Zhang WL, Meng HZ, Yang RF, Yang MW, Sun GH, Liu JH, Shi PX, Liu F and Yang B: Melatonin suppresses autophagy in type 2 diabetic osteoporosis. Oncotarget 7: 52179-52194, 2016.

6. Tanaka T, Latorre MRDO, Jaime PC, Florindo AA, Pippa MG and Zerbini CA: Risk factors for proximal femur osteoporosis in men aged 50 years or older. Osteoporos Int 12: 942-949, 2001

7. Cosman F, de Beur SJ, LeBoff MS, Lewiecki EM, Tanner B, Randall S, Lindsay R; National Osteoporosis Foundation: Clinician's guide to prevention and treatment of osteoporosis. Osteoporos Int 25: 2359-2381, 2014.

8. Arnao MB and Hernández-Ruiz J: Functions of melatonin in plants: A review. J Pineal Res 59: 133-150, 2015.

9. Pinto AR, da Silva NC and Pinato L: Analyses of melatonin, cytokines, and sleep in chronic renal failure. Sleep Breath 20: 339-344, 2016.

10. Tocher DR, Mourente G, Van Der Eecken A, Evjemo JO, Diaz E, Bell JG, Geurden I, Lavens P and Olsen Y: Effects of dietary vitamin $\mathrm{E}$ on antioxidant defence mechanisms of juvenile turbot (Scophthalmusmaximus L.), halibut (Hippoglossushippoglossus L.) and sea bream (Sparusaurata L.). Aquacult Nutr 8: 195-207, 2002.

11. Maes M, Ruckoanich P, Chang YS, Mahanonda N and Berk M: Multiple aberrations in shared inflammatory and oxidative \& nitrosative stress (IO\&NS) pathways explain the co-association of depression and cardiovascular disorder (CVD), and the increased risk for CVD and due mortality in depressed patients. Prog Neuropsychopharmacol Biol Psychiatry 35: 769-783, 2011.
12. Tang X, Rong G, Bu Y, Zhang S, Zhang M, Zhang J and Liang X Advanced oxidation protein products induce hypertrophy and epithelial-to-mesenchymal transition in human proximal tubular cells through induction of endoplasmic reticulum stress. Cell Physiol Biochem 35: 816-828, 2015.

13. Zhao XQ, Liang B, Liu Y and Huang XQ: Agaricoglycerides protect against hepatic ischemia/reperfusion injury by attenuating inflammatory response, oxidative stress, and expression of NF- $\kappa$ B. Evid Based Complement Alternat Med 2015: 142736 , 2015.

14. Izadyar S, Golbarg S, Takavar A and Zakariaee SS: The effect of the lumbar vertebral malpositioning on bone mineral density measurements of the lumbar spine by dual-energy X-ray absorptiometry. J Clin Densitom 19: 277-281, 2016.

15. Norouzi J, Yadollahpour A, Mirbagheri SA, Mazdeh MM and Hosseini SA: Predicting renal failure progression in chronic kidney disease using integrated intelligent fuzzy expert system. Comput Math Methods Med 2016: 6080814, 2016.

16. Pedersen BK and Febbraio M: Exercise and interleukin- 6 action. Expert Rev Endocrinol Metab 1: 319-321, 2014.

17. Yang L, Zhang J and Wang G: The effect of sodium hyaluronate treating knee osteoarthritis on synovial fluid interleukin-1 $\beta$ and clinical treatment mechanism. Pak J Pharm Sci 28 (Suppl 1): 407-410, 2015.

18. Yoo YM, Han TY and Kim HS: Melatonin suppresses autophagy induced by clinostat in preosteoblast MC3T3-E1 cells. Int J Mol Sci 17: 526, 2016.

19. Zong Y, Chen J, Guo J-Z, Zhang X-J, Zhang T-J and Sun L: Resveratrol inhibits lipopolysaccharide-induced activation of osteoclast precursor Raw 264.7 cells. Acta Anatomica Sinica 46: 51-56, 2015. doi: 10.16098/j.issn.0529-1356.2015.01.009.

20. Nurdiana N, Mariati N, Noorhamdani N, Setiawan B, Budhiparama N and Noor Z: Effects of Labisia pumila, on oxidative stress in rat model of post-menopausal osteoporosis. Asian Pac J Reprod 5: 391-394, 2016.

This work is licensed under a Creative Commons Attribution-NonCommercial-NoDerivatives 4.0 International (CC BY-NC-ND 4.0) License. 